\title{
Differential expression of the Notch1 receptor, and its ligands Dll1, Dll3 and Dll4 in distinct human pituitary adenoma subtypes
}

\author{
JIANFU ZHANG ${ }^{1,2}$, HUA GAO $^{1}$ and YAZHUO ZHANG ${ }^{1}$ \\ ${ }^{1}$ Beijing Neurosurgical Institute, Capital Medical University, Beijing 100050; \\ ${ }^{2}$ Neurosurgical Department, Weihai Municipal Hospital, Huancui, Weihai 264200, P.R. China
}

Received August 20, 2015; Accepted February 7, 2017

DOI: $10.3892 / 01.2017 .5997$

\begin{abstract}
Pituitary adenoma (PA) is a common type of benign tumor of the pituitary gland that is characterized by specific signs and symptoms, primarily associated with hypersecretion of pituitary glycoprotein hormones (thyroid-stimulating, growth and adrenocorticotrophic hormones, and prolactin). Surgery is the first-line treatment, although postoperative residual tissues/cells and subsequent recurrence remain notable complications. Gene therapy is an effective approach for treatment, as previous studies have demonstrated that the Notch signaling pathway participates in the pathogenesis of PA. The focus of the present study was on the associations between the expression of the Notch1 receptor and its ligands $\delta$-like canonical Notch ligand (Dll) 1, Dll3 and Dll4 in patients with PA. Using reverse transcription-quantitative polymerase chain reaction and western blot analyses, to the best of our knowledge, this is the first study to provide a description of the differential expression of the Notch1 receptor and its ligands Dl11, Dll3, and Dll4 in various types of human PA at the mRNA and protein levels. The results of the present study demonstrated that Notch1 protein expression was positively correlated with Dll4 protein expression, but negatively correlated with Dll3 protein expression, indicating synergistic effects between the Notch1 receptor and Dl14 ligand. Furthermore, the Dl13 ligand may be an inhibitor of the Notch1 receptor, indicating an antagonistic association between Notch1 and the Dll3 ligand. These results have identified a potential target for the treatment of patients with PA.
\end{abstract}

\section{Introduction}

Pituitary adenoma (PA) accounts for between 10 and $25 \%$ of all types of intracranial neoplasm, with an estimated prevalence in

Correspondence to: Professor Yazhuo Zhang, Beijing Neurosurgical Institute, Capital Medical University, 6 Tiantan Xili, Dongcheng, Beijing 100050, P.R. China

E-mail: zyz2004520@yeah.net

Key words: Notch signaling pathway, notch1, $\delta$-like canonical Notch ligand-1, $\delta$-like canonical Notch ligand-3, $\delta$-like canonical Notch ligand-4, pituitary adenoma, pathogenesis the general population of $\sim 17 \%$ (1). PA may develop at any age, but primarily occurs at between 30 and 40 years old, with an equal prevalence among males and females. Pathologically, PA is classified into the following subtypes: Non-functioning (NF), growth hormone $(\mathrm{GH})$-secreting, prolactin (PRL)-secreting, thyroid-stimulating hormone (TSH)-secreting and adrenocorticotrophic hormone (ACTH)-secreting $(2,3)$. Furthermore, NFPA is classified into null cell adenoma, which does not secrete hormones and gonadotroph adenoma (GA), which secretes luteinizing hormone ( $\mathrm{LH})$, follicle-stimulating hormone (FSH), and their subunits (4). Currently, surgery is the first-line treatment for patients with PA.

Postoperative residual tissues/cells and subsequent recurrence remain problematic (5-7). However, gene therapy presents an effective approach to solve this problem. Previous studies have demonstrated that mitochondrial dysfunction, oxidative stress, cell-cycle dysregulation and abnormal mitogen-activated protein kinase signaling are significantly associated with the occurrence of PA (8-10). These studies provide novel insights into the underlying molecular mechanism of human PA pathogenesis, and provide opportunities for in-depth investigations on PA and gene therapy discovery (8-10).

The Notch signaling pathway is a short-range communication transducer that is involved in the regulation of numerous cellular processes, such as cell fate, maintenance of stem cells, apoptosis during development and renewal of adult tissue (11-13). In humans, there are four known Notch receptors (Notch1, 2, 3 and 4), two Jagged ligands (Jagged 1 and 2) and three $\delta$-like canonical Notch ligands [(Dll) 1, Dll3 and D114] (14). The Notch receptor is activated by its ligands, and the intracellular domain of Notch (Notch-IC) is separated by $\alpha$ - and $\gamma$-secretase prior to entry into the nucleus. Binding of Notch-IC to a ubiquitous transcription factor activates the transcription of Notch-targeted genes $(12,13,15)$. Notch-ligand interactions participate in the pathogenesis of a number of human diseases, including the formation and progression of PA. A study conducted by Moreno et al (16) revealed that Notch 3 was overexpressed in human NFPA, but not in GH- and PRL-secreting adenoma. In addition, this was coupled with the downregulation of D111 ligand expression, which was identified through gene expression profiling, reverse transcription-quantative polymerase chain reaction (RT-qPCR) and proteomic analyses. Another previous study demonstrated a significant upregulation of Notch3 mRNA and protein expression in 
NFPA (17). In our previous study, RT-qPCR and western blot analyses demonstrated that the upregulation of the Notch3 receptor and Jagged1 ligand occurs in human NFPA, but not in normal human PA or hormone-secreting adenoma (18). However, the expression levels and functions of the other Notch receptors, and ligands in PA remain to be reported. In the present study, the role of Notch1, and its ligands D111, Dll3 and Dll4, was investigated in various types of PA. To the best of our knowledge, the present study is the first to describe the associations between the differential expression of Notch1, Dl11, Dll3 and D114. This may aid in the development of gene therapies for treatment of patients with PA.

\section{Materials and methods}

Patients and tissue samples. A total of 16 PA tissue samples were collected from patients who underwent endoscopic transsphenoidal surgery at the Beijing Tiantan Hospital (Beijing, China). Written informed consent was obtained from all patients and the study protocol was approved by the Ethics Committee of Beijing Tiantan Hospital. All samples were rinsed in sterile saline, snap-frozen in liquid nitrogen and subsequently stored in liquid nitrogen until required for analysis. Clinicopathological characteristics of the patients are summarized in Table I. Individual PA samples were classified on the basis of the profile of adenohypophyseal hormone content by histological and immunohistochemical analyses prior to molecular analysis.

Protein preparation and western blot analysis. The resected PA samples were thawed and homogenized in lysis buffer (Abcam., Cambridge, MA, USA) using a handheld microtissue homogenizer. The homogenate was subsequently centrifuged at $12,000 \mathrm{x} g$ for $15 \mathrm{~min}$ at $4^{\circ} \mathrm{C}$ and the supernatant was denatured for $5 \mathrm{~min}$ at $95^{\circ} \mathrm{C}$ in loading buffer. Protein concentrations were measured using the bicinchoninic acid protein assay with bovine serum albumin (Sigma-Aldrich; Merck KGaA, Darmstadt, Germany) as the standard control. Total proteins $(60 \mu \mathrm{g})$ were separated using SDS-PAGE on 8 or $10 \%$ gels, subsequently transferred onto nitrocellulose membranes and incubated with 5\% non-fat milk in Tris-buffered saline containing Tween-20 (TBST) for $1 \mathrm{~h}$ at room temperature. Membranes were then probed overnight with the corresponding primary antibody (Ab) at $4^{\circ} \mathrm{C}$, followed by three 10 -min washes with TBST. Subsequently, membranes were incubated with horseradish peroxidase-conjugated secondary Abs at room temperature for $1 \mathrm{~h}$. The following rabbit primary Abs were used: Monoclonal Notch1 (dilution, 1:2,000; catalog no., ab52627; Abcam, ), polyclonal DLL1 (dilution, 1:2,000; catalog no., ab84620; Abcam), polyclonal DLL3 (dilution, 1:1,000; catalog no., ab63707; Abcam) and polyclonal DLL4 (dilution, 1:2,000; catalog no., ab7280; Abcam). Goat anti-rabbit IgG H\&L (horseradish peroxidase) secondary antibody was used (dilution, 1:5,000; catalog no., ab6721; Abcam). An enhanced chemiluminescence system (GE Healthcare Life Sciences, Chalfont, UK) was used according to the manufacturer's protocol in order to visualize the positive bands on transparent medical X-ray film. The final data were subjected to grayscale scanning and semi-quantitative analysis using Quantity One software version 4.6.2 (Bio-Rad Laboratories, Inc., Hercules, CA, USA).
RNA extraction and qPCR analysis. Total RNA was extracted from frozen PA samples (40-60 mg) using TRIzol ${ }^{\circledR}$ reagent (Invitrogen; Thermo Fisher Scientific, Inc., Waltham, MA, USA) and first-strand cDNA was synthesized from total RNA using the SuperScript ${ }^{\mathrm{TM}}$ First-Strand Synthesis system with SuperScript II reverse transcriptase (Invitrogen; Thermo Fisher Scientific, Inc.), according to the manufacturer's protocol. qPCR was performed in an Applied Biosystems 7500 Fast Real-Time PCR system (Applied Biosystems; Thermo Fisher Scientific, Inc.) using Platinum SYBR-Green/ROX qPCR Supermix-UDG (Invitrogen; Thermo Fisher Scientific, Inc.). qPCR was performed using a $25-\mu 1$ reaction volume containing 2X Master mix (12.5 $\mu \mathrm{l})$, forward/reverse primers (0.5 $\mu \mathrm{l}$ each, $10 \mu \mathrm{M}$; Table II), sample cDNA ( $1 \mu \mathrm{l})$, and double distilled water $(10.5 \mu \mathrm{l})$. The thermocycling conditions were as follows: $50^{\circ} \mathrm{C}$ for $120 \mathrm{sec}, 95^{\circ} \mathrm{C}$ for $120 \mathrm{sec}$, followed by 40 cycles at $95^{\circ} \mathrm{C}$ for $15 \mathrm{sec}$ and $60^{\circ} \mathrm{C}$ for $30 \mathrm{sec}$. Fluorescence of the PCR products was measured following completion of the extension step. mRNA expression levels of the genes of interest were determined from the threshold cycle $(\mathrm{Cq})$ and the relative expression levels of the genes examined were normalized relative to that of GAPDH and quantified using the $2^{-\Delta \Delta \mathrm{Cq}}$ method (7500 software version 2.3; Thermo Fisher Scientific, Inc.) (19).

Statistical analysis. All data are presented as the mean \pm standard error. Statistical analyses of protein expression between tumor types were performed using Student's t-test or non-parametric Mann-Whitney U test. Correlations were identified using Pearson's rank-sum test. $\mathrm{P}<0.05$ was considered to indicate a statistically significant difference. All analyses were performed using SPSS software (version 17.0; SPSS Inc., Chicago, IL, USA).

\section{Results}

Tumor classification. The clinicopathological characteristics of the 16 adenoma tissue samples used in the present study are listed in Table I. There were 9 male and 7 female patients. The mean patient age was 43.3 years (range, 29-63 years) and the mean tumor diameter was $2.6 \mathrm{~cm}$ (range, $1.5-4.3 \mathrm{~cm}$ ). There were 8 NFPA, 4 GH-secreting and 4 PRL-secreting adenoma samples. The 4 NFPA samples were identified to be anterior pituitary hormone-negative using immunohistochemical analysis and were designated as NF- tumors. The remaining 4 NFPA samples were stained with LH and/or FSH, and designated immunohistochemically positive (NF+). The 4 PRL-secreting adenoma samples manifested as hyperprolactinemia, whereas the $4 \mathrm{GH}$-secreting adenoma samples were characterized as acromegaly. For the 8 patients with NFPA, headache and visual defects were the main symptoms.

Western blot analysis. Western blot analysis demonstrated that Notch1 protein expression (Fig. 1A) was highest in GA and GH-secreting adenoma samples, as compared with null cell and PRL-secreting adenoma samples. Dll1 protein expression (Fig. 1B) was increased in GH-secreting and PRL-secreting adenoma samples, as compared with null cell adenoma and GA $(\mathrm{P}<0.05)$. The highest level of Dll3 protein expression 
Table I. Clinicopathological characteristics of 16 patients with pituitary adenoma.

\begin{tabular}{cccclc}
\hline Patient ID & Sex & $\begin{array}{c}\text { Age, } \\
\text { years }\end{array}$ & $\begin{array}{c}\text { Tumor } \\
\text { size, cm }\end{array}$ & \multicolumn{1}{c}{$\begin{array}{c}\text { Clinical characteristic } \\
\text { Immunohistochemical } \\
\text { analysis }\end{array}$} \\
\hline 1 & M & 43 & 2.8 & Headache and hypopituitarism & NF- \\
2 & F & 50 & 4.3 & Headache and visual defects & NF- \\
3 & M & 57 & 2.0 & Headache & NF- \\
4 & M & 46 & 3.5 & Visual defects & NF+: LH+, FSH+ \\
5 & F & 42 & 1.7 & Headache & NF+: FSH+ \\
6 & M & 32 & 3.6 & Headache and visual defects & NF+: FSH+ \\
7 & F & 53 & 3.0 & Visual defects & GH+ \\
8 & M & 45 & 2.1 & Symptomless & GH+ \\
9 & M & 31 & 1.5 & Acromegaly & GH+ \\
10 & F & 47 & 1.6 & Acromegaly & GH+ \\
11 & M & 39 & 2.0 & Acromegaly & PRL+ \\
12 & F & 29 & 3.1 & Acromegaly & PRL+ \\
13 & F & 63 & 3.4 & Hyperprolactinemia & PRL+ \\
15 & M & 29 & 2.2 & Hyperprolactinemia & PRL+ \\
\hline 16 & M & 43 & 1.9 & Hyperprolactinemia & Hyperprolactinemia \\
\hline
\end{tabular}

M, male; F, female; PRL, prolactin; NF, non-functioning; FSH, follicle-stimulating hormone; GH, growth hormone; LH, luteinizing hormone.

Table II. Quantitative polymerase chain reaction primers.

\begin{tabular}{lclll}
\hline Gene name & $\begin{array}{c}\text { Product } \\
\text { size, bp }\end{array}$ & \multicolumn{1}{c}{ Forward sequence $\left(5^{\prime}-3^{\prime}\right)$} & Reverse sequence $\left(5^{\prime}-3^{\prime}\right)$ & Temperature, ${ }^{\circ} \mathrm{C}$ \\
\hline Notch1 & 172 & AAGCTGCATCCAGAGGCAAAC & TGGCATACACACTCCGAGAACAC & 64.0 \\
DLL1 & 112 & GATGTGATGAGCAGCATGGA & CCATGGAGACAGCCTGGATA & 60.8 \\
DLL3 & 205 & AATCGCCCTGAAGATGTAGACC & GCACCACCGAGCAAATACAA & 61.70 \\
DLL4 & 115 & GGCCAACTATGCTTGTGAATGTC & ACCTCGGTTCAGGCACTGTC & 63.0 \\
\hline
\end{tabular}

(Fig. 1C) occurred in null cell adenoma and the lowest level was identified in PRL-secreting adenoma. Significant differences were identified between any two groups $(\mathrm{P}<0.05)$. Dll4 protein expression (Fig. 1D) was highest in GH-secreting adenoma and PRL-secreting adenoma, and lowest in null cell adenoma. As a group, NFPA demonstrated lower D114 expression levels, compared with GH-secreting and PRL-secreting adenoma samples $(\mathrm{n}=4 ; \mathrm{P}<0.05)$.

qPCR analysis. Notch1 mRNA expression (Fig. 2A) was highest in GA and there were significant differences compared with the other three groups $(\mathrm{P}<0.05)$. Dll1 mRNA expression (Fig. 2B) was lowest in GH-secreting adenoma, whereas no significant differences were identified between the other three groups ( $\mathrm{P}>0.05$ ). Dll3 mRNA expression (Fig. 2C) was highest in GA and lowest in GH-secreting adenoma. Significant differences compared with the other three groups were identified $(\mathrm{P}<0.05)$. D114 mRNA expression (Fig. 2D) was lowest in GA and there were significant differences compared with the other three groups $(\mathrm{P}<0.05)$. No significant differences were identified between the other three groups $(\mathrm{P}>0.05)$.
Associations between the expression levels of Notch1, and Dll1, Dll3 and Dll4. Notch1 protein expression was demonstrated to be positively associated with Dll4 protein expression (Fig. 3A), with a Pearson's correlation coefficient of 0.825 $(\mathrm{P}=0.016)$. However, Notch1 protein expression was identified to be negatively correlated with Dll3 protein expression (Fig. 3B), with a Pearson's correlation coefficient of -0.703 $(\mathrm{P}=0.012)$. No significant association was identified between Notch1 and Dll1 protein expression.

\section{Discussion}

The Notch signaling pathway is highly conserved among the majority of multicellular organisms, and is used to determine cell fates and regulate pattern formation, whereas its dysfunction results in a variety of developmental defects, and adult pathologies (20). The Notch signaling pathway may convey antitumor or tumor-promoting effects in different tumor types depending on the microenvironment. Thus, inhibition of the Notch signaling pathway may either inhibit or promote tumor growth $(21,22)$. 

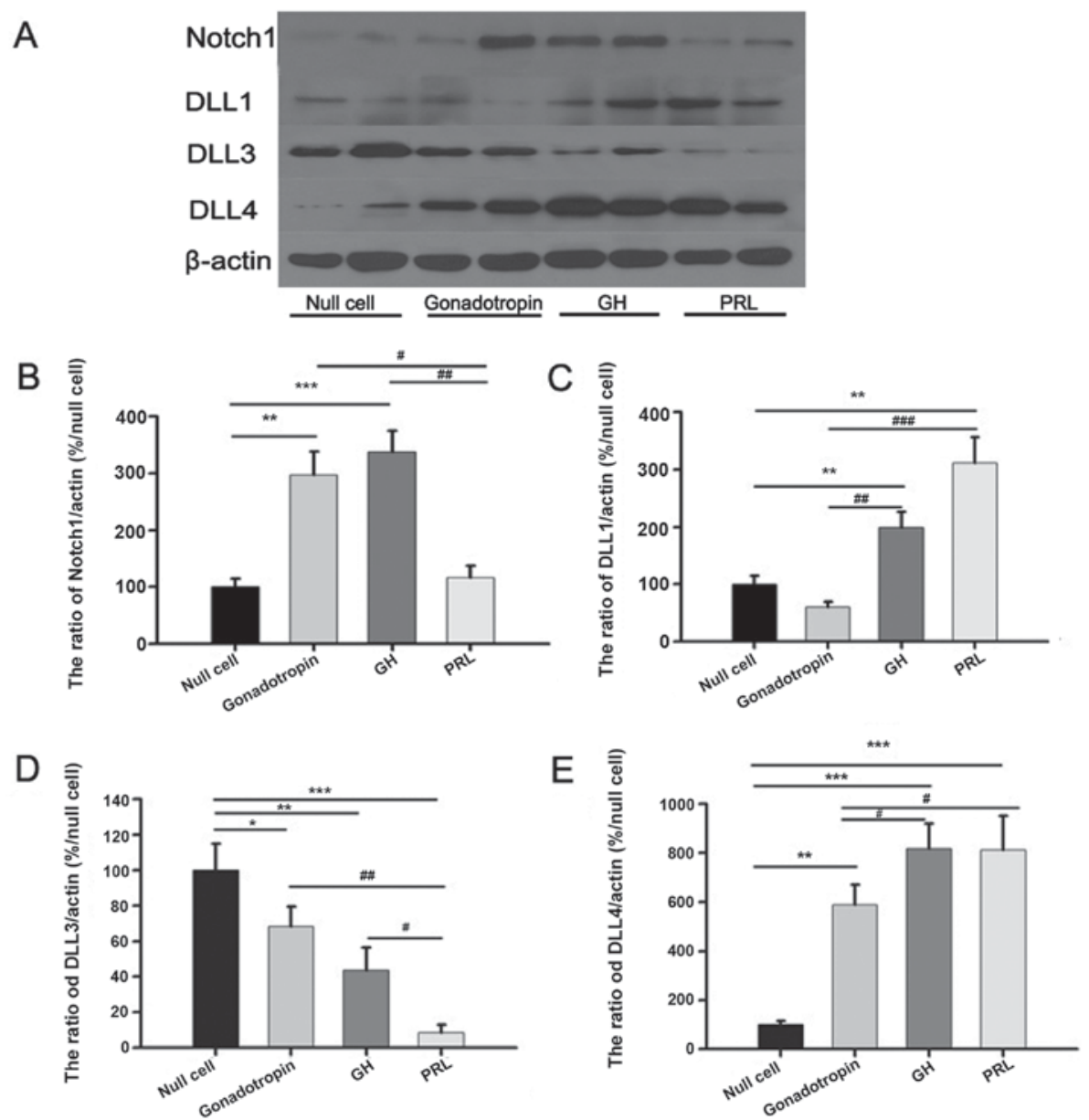

Figure 1. Western blot analysis of tissue samples from patients with pituitary adenoma. (A) Representative western blot analysis of Notch1, Dll1, Dl13 and Dl14 protein expression. Quantification of (B) Notch1, (C) Dll1, (D) Dll3 and (E) Dll4 protein expression in null cell adenoma (n=4), GA (n=4), GH-secreting (n=4) and PRL-secreting adenoma $(n=4)$. $\beta$-actin was used for normalization. Western blot analysis revealed increased Notch1 expression in GA and GH-secreting adenoma $(\mathrm{P}=0.023)$. Increased $\mathrm{Dll1}$ expression was demonstrated in $\mathrm{GH}$-secreting and $\mathrm{PRL}$-secreting adenoma $(\mathrm{P}=0.036)$. Increased Dll3 expression was demonstrated in null cell adenoma $(\mathrm{P}=0.041)$. Decreased Dll3 expression was evident in PRL-secreting adenoma ( $\mathrm{P}=0.037)$. Increased Dll4 expression was detected in GH-secreting and PRL-secreting adenoma $(\mathrm{P}=0.030)$. Decreased Dll4 expression was evident in null cell adenoma $(\mathrm{P}=0.016)$. Dll, $\delta$-like canonical Notch ligand; GH, growth hormone; PRL, prolactin; GA, gonadotroph adenoma. ${ }^{*}$ Comparison between null cell and the other groups; "comparison between the three groups except null cell.

Abundant evidence indicates that Notch1 is involved in the angiogenesis and tumorigenesis of various types of malignancy. It has been revealed previously that Notch1 signaling is the convergence point of numerous signaling pathways (23). The dysfunction of Notch1 may inhibit cell differentiation, resulting in the malignant transformation of undifferentiated cells. In addition, Notch1 has been reported to induce cell cycle arrest and apoptosis in certain tumors, and may induce epithelial-mesenchymal transition, which is consistent with the cancer stem cell phenotype of pancreatic cancer cells (24). Previous research has demonstrated that Notch1 has different functions in the various phases of tumor development. Notch1 serves a promoting role in the majority of carcinoma types, but serves an antitumor role in skin, lung, liver, thyroid and breast cancer. Notch1 has a promoting effect in the early stage of cervical cancer, while inhibiting tumor growth in end-stage cervical cancer (25). A possible explanation for the differences in Notch1 function may be that it is highly conserved during biological evolution and expressed in various tissues and cells. Furthermore, crosstalk between Notch1 and other signaling pathways may give rise to the diversity and complexity of Notch1 function (26).
The results of the present study provide evidence of Notch1 expression in human PA. Notch1 expression was demonstrated to be increased in GA and GH-secreting adenoma, as compared with null cell and PRL-secreting adenoma, indicating that Notch1 can stimulate and inhibit tumor growth, and hormone production of GA and, GH-secreting adenoma, although the underlying molecular mechanism remains unclear. However, ACTH- and TSH-secreting adenoma were not investigated in the present study.

In a study by Gao et al (27), DLL1 overexpression was observed in hepatic carcinoma, but not in healthy liver tissue. Increased DLL1 expression was able to increase the proliferative ability of hepatic carcinoma, whereas decreased DLL1 expression may decrease this proliferative ability, indicating the tumor-promoting effect of DLL1 in hepatic carcinoma. Another study identified overexpression of Notch1 and DLL1 mRNA, and protein levels in six human glioma cell lines and brain glioma (28). Similarly, Somasundaram et al (29) demonstrated DLL1 overexpression in diffuse astrocytoma, anaplastic astrocytoma and secondary glioblastoma multiforme. Previous studies have demonstrated that D111 expression was increased in GH-secreting and PRL-secreting 
A

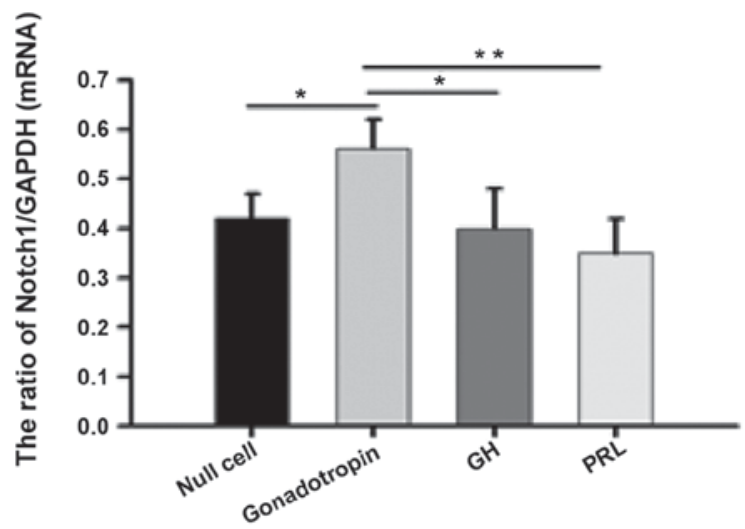

C

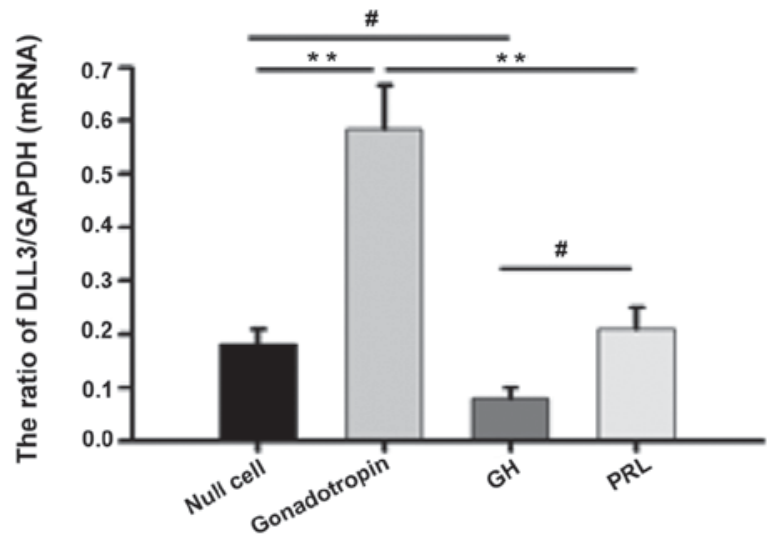

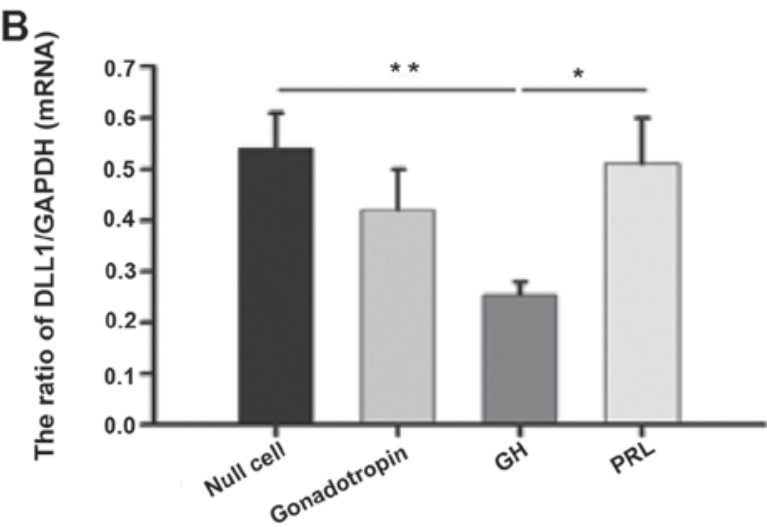

D

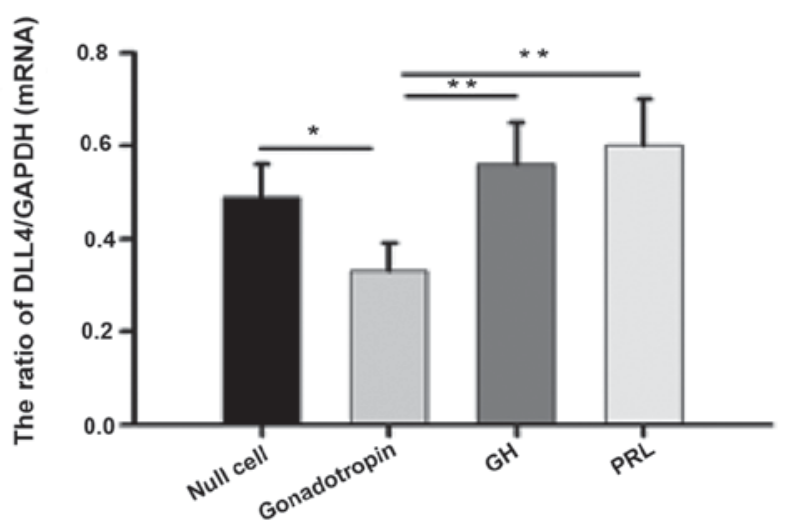

Figure 2. qPCR analysis results of tissue samples from patients with pituitary adenoma. (A) qPCR analysis of relative Notch1 mRNA expression in null cell adenoma $(n=4)$, GA $(n=4), G H-s e c r e t i n g ~(n=4)$ and PRL-secreting adenoma $(n=4)$. Increased Notch1 expression was demonstrated in GA $(P=0.048)$. (B) qPCR analysis of relative Dll1 mRNA expression in null cell adenoma, GA, GH-secreting and PRL-secreting adenoma samples. Dll1 expression was lowest in GH-secreting adenoma $(\mathrm{P}=0.036)$. (C) qPCR analysis of relative Dll3 mRNA expression in null cell adenoma, GA, GH-secreting and PRL-secreting adenoma. Increased D113 expression was demonstrated in GA $(\mathrm{P}=0.028)$. Decreased Dl13 expression was evident in $\mathrm{GH}-\mathrm{secreting}$ adenoma $(\mathrm{P}=0.045)$. (D) qPCR analysis of relative Dl14 mRNA expression in null cell adenoma, GA, GH-secreting and PRL-secreting adenoma. Decreased Dll4 expression was evident in GA (P=0.047). qPCR, quantitative polymerase chain reaction; Dll, $\delta$-like canonical Notch ligand; GH, growth hormone; PRL, prolactin; GA, gonadotroph adenoma. "Comparison between null cell and the other groups; ${ }^{*}$ comparison between the three groups except null cell.
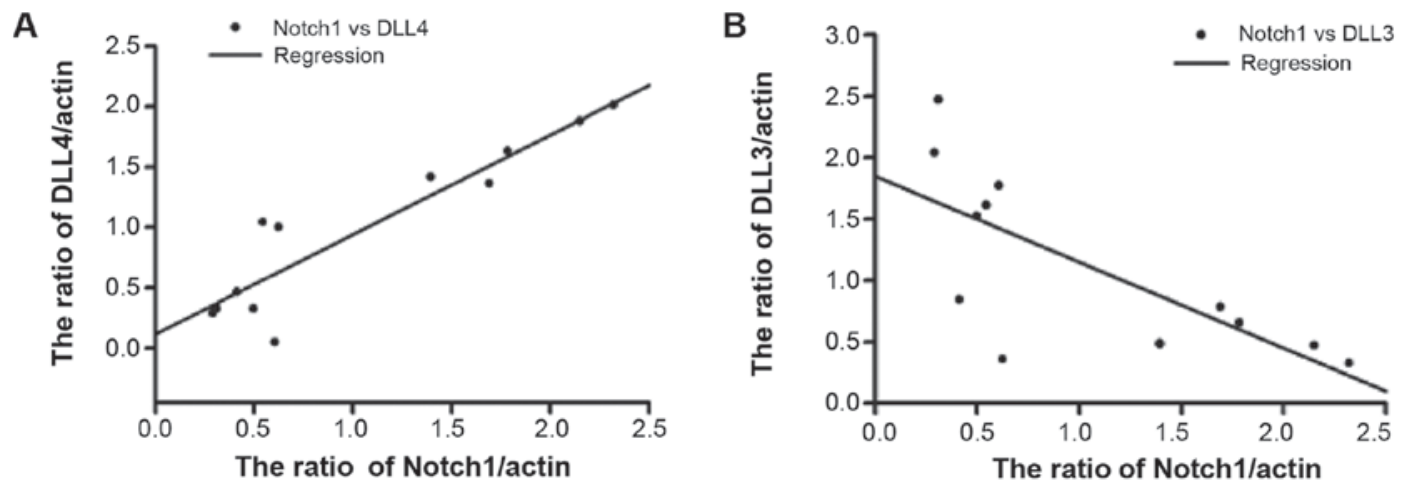

Figure 3. Scatter diagrams demonstrating the associations between (A) Notch1 and DLL4, and between (B) Notch1 and DLL3 following the analysis of tissue samples from patients with pituitary adenoma. Dll, $\delta$-like canonical Notch ligand.

adenoma, compared with null cell adenoma and GA $(17,20)$. Coincidently, the results of the present study demonstrated that DLL1 expression was decreased in NFPA, as compared with GH-secreting adenoma and PRL-secreting adenoma, as previously mentioned. It is hypothesized that Dll1 serves an essential role in the tumorigenesis of $\mathrm{GH}$-secreting and PRL-secreting adenoma.
Dll3 has also been implicated in tumorigenesis and histogenesis. Maemura et al (30) reported that Dll3 was silenced by methylation in human hepatocellular carcinoma and that it inhibits the growth of hepatocellular carcinoma cells. Targeted deletion of D113 in mouse causes a developmental defect in somite segmentation and consequently severe disruption to vertebral formation, resembling spondylocostal dysostosis in 
humans (31). Another study (32) demonstrated that mutations in the Notch1 receptor and D113 ligand caused global disruption to axial segmental patterning, as $30 \%$ of D113-Notch1 double heterozygous animals exhibited localized segmental anomalies similar to congenital vertebral defects in humans. In the present study, it was demonstrated that D113 protein expression was highest in null cell and lowest in PRL-secreting adenoma tissue samples. Significant differences were identified between any two groups. Therefore, Dll3 may be more important in NFPA, including null cell adenoma and GA compared with hormone-secreting adenoma. However, the function and specific underlying molecular mechanism of D113 in PA remain to be elucidated.

As an important ligand of Notch, D114 was demonstrated to be significantly overexpressed in tumor vessels compared with the surrounding blood vessels when it was first detected in a previous study (33). Abundant evidence describes the participation of Dll4 in tumor angiogenesis. A study conducted by Mailhos et al (34) reported that Dll4 expression was elevated in tumor vascular endothelial cells. Tumor hypoxia induces the upregulation of Dll4, which regulates downstream gene expression by activating the D114-Notch signaling pathway to reduce dysfunctional tumor vascular density and promote tumor growth (35-37). Another study (38) concluded that vascular endothelial growth factor (VEGF) upregulates angiogenesis, whereas D114 inhibits VEGF-induced endothelial cell function to prevent excessive blood vessel formation. The results of the present study demonstrated that D114 protein expression was highest in GH- and PRL-secreting adenoma, and lowest in null cell adenoma tissue. As a group, Dll4 protein expression was revealed to be lower in NFPA compared with GH- and PRL-secreting adenoma. These data suggest that Dll4 serves a more important role in the regulation of angiogenesis, and growth of GH- and PRL-secreting adenoma.

To the best of our knowledge, the results of the present study provide the first evidence to demonstrate the differential expression of the Notch1 receptor, and its ligands D111, D113 and Dll4 in various types of human PA at the mRNA and protein level. It was identified that Notch1 protein expression was positively associated with Dl14 protein expression, but negatively associated with D113 protein expression ( $\mathrm{r}=0.815$ and -0.703 , respectively), indicating possible synergistic effects of the Notch1 receptor and its Dll4 ligand. Furthermore, the Dll3 ligand may act as an inhibitor of the Notch1 receptor, indicating an antagonistic association between the two. However, the molecular mechanism underlying the interactions between Notch1 and its ligands remains to be elucidated. In addition, no significant association was identified between the expression levels of the Notch1 receptor and Dll1 ligand.

The results of the present study are consistent with previous studies on other tumors. Previous studies have revealed that the Notch1-DLL4 signaling pathway participates in a range of processes, including the formation, development, invasion and metastasis of malignant tumors (39). Dll4 interacts with Notch1 and associated transcription factors to promote cell proliferation or apoptosis. A previous study identified that Dll4 is an important ligand of the Notch1 signaling pathway and involved in the regulation of tumor angiogenesis (35). The Notch1-DLL4 signaling pathway inhibits excessive vascularization to decrease the number of new blood vessels, whereas in other tumors it accelerates the development of new vessels and maturation to improve the function of the new blood vessels (40).

The results of the present study suggest that there is a potential Notch1-D114 positive feedback loop in PA cells. Dll4 in one cell can bind to Notch1 in a neighboring cell. This interaction results in the proteolytic release of Notch-IC, which translocates to the nucleus, and induces the expression of target genes, including Hes/Hey family genes, cyclin D and nuclear factor- $\kappa \mathrm{B}(41)$. These genes activate the Notch1 signaling pathway in neighboring cells to promote the proliferation of adjacent PA cells. In the present study, Dll4 ligand expression was demonstrated to be upregulated in PA cells, which further upregulates the activation of the Notch1 signaling pathway. Therefore, Notch1 and D114 may promote cell proliferation in PA or induce apoptosis and regulate angiopoiesis via synergistic effects. Therefore, the blockade of the Notch1-DLL4 signaling pathway may serve as a novel gene therapeutic strategy for the treatment of patients with PA. It is plausible that modulators of the Notch signaling pathway, such as $\gamma$-secretase inhibitors developed for Alzheimer's disease, may be useful as pharmacological regulators of $\mathrm{PA}$.

Regarding the association between Notch1 and Dl13, Ladi et al (42) performed multiple assays that demonstrated that Dll3 does not activate Notch signaling, as Notch did not bind to Dll3-expressing cells. However, in a cell-autonomous manner, Dll3 suppressed Notch signaling. Dll3 functions as a dedicated inhibitor of Notch signaling. Chapman et al (31) reported that D113 interacts with Notch1 in the late endocytic compartment and the mechanism for Dll3-mediated cis-inhibition of Notch signaling may involve D113 targeting newly synthesized Notch1 for lysosomal degradation prior to post-translational processing and cell-surface presentation of the receptor. An inhibitory role for D113 in vivo is further supported by the observation of Dll3 protein and Notch1 signaling juxtaposition in the presomitic mesoderm (31).

Following the results of the present study, it is hypothesized that the D113 ligand does not activate Notch1 in PA, but rather functions to autonomously inhibit signaling. The D113 ligand does not bind to Notch1 in neighboring cells and can cis-inhibit ligand-dependent Notch1 activation when expressed on the surface of the same cell as the Notch1 receptor. Dll3 is a potent antagonist of ligand-induced Notch1 signaling when co-expressed with Notch1. It is suggested that a potential Notch1-Dll3 negative feedback loop exists in PA. Therefore, elucidation of the negative feedback mechanism of Notch1 and D113 could aid in guiding the development of novel treatments for patients with PA. However, the underlying molecular mechanism of the interactions between Notch1 and D113/D114 in PA remains to be elucidated.

In conclusion, the results of the present study provide evidence of Notch1, D111, D113 and D114 expression in human PA, indicating that synergistic interactions occur between Notch1 and DLL4 in PA, and the negative correlation between Notch1 and DLL3 expression suggests the presence of a negative feedback loop. Further studies are warranted to clarify the precise mechanism underlying the involvement of the Notch1 signaling pathway in the pathogenesis of PA. 


\section{Acknowledgements}

The present study was supported by the National Natural Science Foundation of China (grant nos. 30971005, 2013BAI09B03 and 201402008).

\section{References}

1. Ezzat S, Asa SL, Couldwell WT, Barr CE, Dodge WE, Vance ML and McCutcheon IE: The prevalence of pituitary adenomas Cancer 101: 613-619, 2004

2. Ironside JW: Best Practice No 172: Pituitary gland pathology. J Clin Pathol 56: 561-568, 2003.

3. Scanarini M and Mingrino S: Functional classification of pituitary adenomas. Acta Neurochir (Wien) 52: 195-202, 1980.

4. Korbonits M and Carlsen E: Recent clinical and pathophysiological advances in non-functioning pituitary adenomas. Horm Res 71 (Suppl 2): S123-S130, 2009.

5. Jaffe CA: Clinically non-functioning pituitary adenoma. Pituitary 9: 317-321, 2006.

6. Daly AF, Rixhon M, Adam C, Dempegioti A, Tichomirowa MA and Beckers A: High prevalence of pituitary adenomas: A cross-sectional study in the province of Liege, Belgium. J Clin Endocrinol Metab 91: 4769-4775, 2006.

7. Greenman Y and Stern N: Non-functioning pituitary adenomas. Best Pract Res Clin Endocrinol Metab 23: 625-638, 2009.

8. Zhan X and Desiderio DM: Signaling pathway networks mined from human pituitary adenoma proteomics data. BMC Med Genomics 3: 13, 2010.

9. Indraccolo S, Minuzzo S, Masiero $M$ and Amadori A: Ligand-driven activation of the notch pathway in T-ALL and solid tumors: Why Not(ch)? Cell Cycle 9: 80-85, 2010.

10. Melmed S: Pathogenesis of pituitary tumors. Nature Rev Endocrinol 7: 257-266, 2011.

11. Bianchi S, Dotti MT and Federico A: Physiology and pathology of notch signalling system. J Cell Physiol 207: 300-308, 2006.

12. Lai EC: Notch signaling: Control of cell communication and cell fate. Development 131: 965-973, 2004.

13. Artavanis-Tsakonas S, Rand MD and Lake RJ: Notch signaling: Cell fate control and signal integration in development. Science 284: 770-776, 1999 .

14. Greenwald I: LIN-12/Notch signaling: Lessons from worms and flies. Genes Dev 12: 1751-1762, 1998.

15. Artavanis-Tsakonas S, Matsuno K and Fortini ME: Notch signaling. Science 268: 225-232, 1995.

16. Moreno CS, Evans CO, Zhan X, Okor M, Desiderio DM and Oyesiku NM: Novel molecular signaling and classification of human clinically nonfunctional pituitary adenomas identified by gene expression profiling and proteomic analyses. Cancer Res 65: 10214-10222, 2005.

17. Miao Z, Miao Y, Lin Y and Lu X: Overexpression of the Notch3 receptor in non-functioning pituitary tumours. J Clin Neurosci 19: 107-110, 2012

18. Lu R, Gao H, Wang H, Cao L, Bai J and Zhang Y: Overexpression of the Notch 3 receptor and its ligand Jagged1 in human clinically non-functioning pituitary adenomas. Oncol Lett 5: 845-851, 2013.

19. Livak KJ and Schmittgen TD: Analysis of relative gene expression data using real-time quantitative PCR and the 2(-Delta Delta C(T)) method. Methods 25: 402-408, 2001.

20. Wang Z, Li Y, Kong D and Sarkar FH: The role of Notch signaling pathway in epithelial-mesenchymal transition (EMT) during development and tumor aggressiveness. Curr Drug Targets 11 745-751, 2010

21. Hu XB, Feng F, Wang YC, Wang L, He F, Dou GR, Liang L, Zhang HW, Liang YM and Han H: Blockade of Notch signaling in tumor-bearing mice may lead to tumor regression, progression, or metastasis, depending on tumor cell types. Neoplasia 11 32-38, 2009

22. Roy M, Pear WS and Aster JC: The multifaceted role of Notch in cancer. Curr Opin Genet Dev 17: 52-59, 2007.

23. Weijzen S, Rizzo P, Braid M, Vaishnav R, Jonkheer SM, Zlobin A, Osborne BA, Gottipati S, Aster JC, Hahn WC, et al: Activation of Notch-1 signaling maintains the neoplastic phenotype in human Ras-transformed cells. Nat Med 8: 979-986, 2002
24. Bao B, Wang Z, Ali S, Kong D, Li Y, Ahmad A, Banerjee S, Azmi AS, Miele L and Sarkar FH: Notch-1 induces epithelial-mesenchymal transition consistent with cancer stem cell phenotype in pancreatic cancer cells. Cancer Lett 307: 26-36, 2011.

25. Chen Y, De Marco MA, Graziani I, Gazdar AF, Strack PR, Miele L and Bocchetta M: Oxygen concentration determines the biological effects of Notch-1 signaling in adenocarcinoma of the lung. Cancer Res 67: 7954-7959, 2007.

26. Koch U and Radtke F: Notch and cancer: A double-edged sword. Cell Mol Life Sci 64: 2746-2762, 2007.

27. Gao J, Chen C, Hong L, Wang J, Du Y, Song J, Shao X, Zhang J, Han H, Liu J and Fan D: Expression of Jagged1 and its association with hepatitis B virus X protein in hepatocellular carcinoma. Biochem Biophys Res Commun 356: 341-347, 2007.

28. Purow BW, Haque RM, Noel MW, Su Q, Burdick MJ, Lee J, Sundaresan T, Pastorino S, Park JK, Mikolaenko I, et al: Expression of Notch-1 and its ligands, Delta-Like-1 and Jagged-1, is critical for glioma cell survival and proliferation. Cancer Res 65: 2353-2363, 2005.

29. Somasundaram K, Reddy SP, Vinnakota K, Britto R, Subbarayan M, Nambiar S, Hebbar A, Samuel C, Shetty M, Sreepathi HK, et al: Upregulation of ASCL1 and inhibition of Notch signaling pathway characterize progressive astrocytoma. Oncogene 24: 7073-7083, 2005.

30. Maemura K, Yoshikawa H, Yokoyama K, Ueno T, Kurose H, Uchiyama K and Otsuki Y: Delta-like 3 is silenced by methylation and induces apoptosis in human hepatocellular carcinoma. Int J Oncol 42: 817-822, 2013.

31. Chapman G, Sparrow DB, Kremmer E and Dunwoodie SL: Notch inhibition by the ligand DELTA-LIKE 3 defines the mechanism of abnormal vertebral segmentation in spondylocostal dysostosis. Hum Mol Genet 20: 905-916, 2011.

32. Loomes KM, Stevens SA, O'Brien ML, Gonzalez DM, Ryan MJ, Segalov M, Dormans NJ, Mimoto MS, Gibson JD, Sewell W, et al: Dll3 and Notch1 genetic interactions model axial segmental and craniofacial malformations of human birth defects. Dev Dyn 236: 2943-2951, 2007.

33. Patel NS, Li JL, Generali D, Poulsom R, Cranston DW and Harris AL: Up-regulation of delta-like 4 ligand in human tumor vasculature and the role of basal expression in endothelial cell function. Cancer Res 65: 8690-8697, 2005.

34. Mailhos C, Modlich U, Lewis J, Harris A, Bicknell R and Ish-Horowicz D: Delta4, an endothelial specific notch ligand expressed at sites of physiological and tumor angiogenesis. Differentiation 69: 135-144, 2001.

35. Li JL, Sainson RC, Shi W, Leek R, Harrington LS, Preusser M, Biswas S, Turley H, Heikamp E, Hainfellner JA and Harris AL: Delta-like 4 Notch ligand regulates tumor angiogenesis, improves tumor vascular function, and promotes tumor growth in vivo. Cancer Res 67: 11244-11253, 2007.

36. Djokovic D, Trindade A, Gigante J, Badenes M, Silva L, Liu R, Li X, Gong M, Krasnoperov V, Gill PS and Duarte A: Combination of D114/Notch and Ephrin-B2/EphB4 targeted therapy is highly effective in disrupting tumor angiogenesis. BMC Cancer 10: 641, 2010.

37. Al Haj Zen A, Oikawa A, Bazan-Peregrino M, Meloni M, Emanueli C and Madeddu P: Inhibition of delta-like-4-mediated signaling impairs reparative angiogenesis after ischemia. Circ Res 107: 283-293, 2010.

38. Williams CK, Li JL, Murga M, Harris AL and Tosato G: Up-regulation of the Notch ligand Delta-like 4 inhibits VEGF-induced endothelial cell function. Blood 107: 931-939, 2006.

39. Weng AP and Aster JC: Multiple niches for Notch in cancer: Context is everything. Curr Opin Genet Dev 14: 48-54, 2004.

40. Hellström M, Phng LK, Hofmann JJ, Wallgard E, Coultas L, Lindblom P, Alva J, Nilsson AK, Karlsson L, Gaiano N, et al: Dl14 signalling through Notch1 regulates formation of tip cells during angiogenesis. Nature 445: 776-780, 2007.

41. Bray SJ: Notch signalling: A simple pathway becomes complex. Nat Rev Mol Cell Biol 7: 678-689, 2006.

42. Ladi E, Nichols JT, Ge W, Miyamoto A, Yao C, Yang LT, Boulter J, Sun YE, Kintner C and Weinmaster G: The divergent DSL ligand D113 does not activate Notch signaling but cell autonomously attenuates signaling induced by other DSL ligands. J Cell Biol 170: 983-992, 2005. 